\title{
Geotecnologias aplicadas à avaliação de parâmetros biofísicos do Pantanal
}

\author{
Ricardo Guimarães Andrade ${ }^{(1)}$, Gilberto Chohaku Sediyama(2), Adriano Rolim da Paz ${ }^{(3)}$, \\ Evaldo de Paiva Lima ${ }^{(4)}$ e Alexandro Gomes Facco(5)
}

\begin{abstract}
(1)Embrapa Monitoramento por Satélite, Avenida Soldado Passarinho, no 303, Fazenda Chapadão, CEP $13070-115$ Campinas, SP. E-mail: ricardo@cnpm.embrapa.br (2)Universidade Federal de Viçosa, Departamento de Engenharia Agrícola, CEP 36570-000 Viçosa, MG. E-mail: g.sediyama@ufv.br (3)Universidade Federal da Paraíba, Cidade Universitária, CEP 58051-900 João Pessoa, PB. E-mail: adrianorpaz@yahoo.com.br (4)Embrapa Solos, Jardim Botânico, CEP 22460-000 Rio de Janeiro, RJ. E-mail: evaldo@cnps.embrapa.br ${ }^{(5)}$ Universidade Federal do Espírito Santo, Centro Universitário Norte do Espírito Santo, Departamento de Ciências Agrárias e Biológicas, CEP 29932-540 São Mateus, ES. E-mail: agfacco@ceunes.ufes.br
\end{abstract}

Resumo - O objetivo deste trabalho foi avaliar parâmetros biofísicos de superfície do Bioma Pantanal com a aplicação de geotecnologias. Foram utilizados o algoritmo Sebal ("surface energy balance algorithm for land"), imagens do sensor Modis ("moderate-resolution imaging spectroradiometer") e o mapa de classes de uso e cobertura da terra. Os resultados obtidos para NDVI, temperatura da superfície, albedo, fluxo de calor sensível diário, saldo de radiação diário e evapotranspiração real diária foram consistentes com dados de literatura para os diferentes usos e cobertura da terra, e corroboram a eficiência da capacidade analítica e sinóptica das estimativas do Sebal. Tais resultados mostram o potencial de geotecnologias na implementação de modelos ou algoritmos voltados para a compreensão da dinâmica de processos biofísicos de interação solo-planta-atmosfera do Pantanal.

Termos para indexação: balanço de energia, ciclo hidrológico, interação solo-planta-atmosfera, Sebal, uso e cobertura da terra.

\section{Geotechnologies applied to the assessment of biophysical parameters of the Pantanal biome}

\begin{abstract}
The objective of this work was to evaluate surface biophysical parameters of the Pantanal biome, Brazil, by the application of geotechnologies. The Sebal algorithm, Modis imagery, and land use and land cover map were used. The obtained results for NDVI, surface temperature, albedo, daily sensible heat flux, daily net radiation and daily actual evapotranspiration were consistent with literature data for the different land use and land cover classes, and corroborate the analytical and synoptic capacity of Sebal estimates. These results show the potential of geotechnologies in the implementation of models or algorithms developed for the understanding of biophysical process dynamics related to the soil-plant-atmosphere interactions in Pantanal.
\end{abstract}

Index terms: energy balance, hydrological cycle, soil-plant-atmosphere interaction, Sebal, land use and land cover.

\section{Introdução}

Vêm crescendo as pressões econômicas, políticas e sociais, para que o aumento da produtividade na região pantaneira seja compatibilizado com a conservação do Pantanal (Santos \& Costa, 2002). Esse bioma tem a maior área contínua inundável do planeta, com ciclo de alagamento anual que abrange aproximadamente $30 \%$ do seu território.

O regime de inundações é o fator ecológico fundamental do Bioma Pantanal, que determina os pulsos de seus principais processos bióticos e abióticos, e as composições específicas das unidades de paisagem (Adami et al., 2008). Além disso, os períodos de seca e cheia influenciam significativamente o balanço de energia e o ciclo hidrológico regional.

Estudos relacionados à demanda hídrica em escala regional, que identificam variabilidades espaciais e temporais, são fundamentais no diagnóstico e gerenciamento de bacias hidrográficas. No entanto, em razão da escassez de dados meteorológicos de algumas regiões e da dificuldade de obtenção, de forma representativa, de alguns parâmetros biofísicos de superfície em escala regional, as técnicas de

Pesq. agropec. bras., Brasília, v.47, n.9, p.1227-1234, set. 2012 
sensoriamento remoto têm sido imprescindíveis para a obtenção de informações de áreas extensas de forma rápida e efetiva (Compaoré et al., 2008).

Alguns estudos têm sido realizados com o objetivo de determinar a evapotranspiração (ET) com base na equação do balanço de energia à superfície e em técnicas de sensoriamento remoto (Bastiaanssen et al., 1998a, 1998b; Bastiaanssen, 2000; Tasumi et al., 2008). Para a obtenção da ET a partir de imagens orbitais, são utilizados algoritmos e modelos. Entre eles, o Sebal ('surface energy balance algorithm for land') tem-se destacado em estudos que envolvem estimativas dos componentes do balanço de energia em áreas extensas, com possibilidade de ser aplicado com poucos dados coletados de superfície.

O objetivo deste trabalho foi avaliar alguns parâmetros biofísicos de superfície do Bioma Pantanal com a aplicação de geotecnologias.

\section{Material e Métodos}

A área de estudo foi representada pelo Bioma Pantanal, que abrange os Estados do Mato Grosso e Mato Grosso do Sul. O Pantanal tem área de $150.355 \mathrm{~km}^{2}$ (Abdon et al., 2007) e sofre influência biogeográfica dos biomas vizinhos, como o Cerrado a leste, a Amazônia ao norte, e o Chaco a sudoeste (Figura 1). A planície aluvionar do Pantanal é formada principalmente por tributários da margem esquerda do Rio Paraguai, no território brasileiro, com sua borda oeste tocando o território da Bolívia ao norte, e o do Paraguai ao sul. A altitude na planície varia, em média, de 60 a $150 \mathrm{~m}$. No Pantanal, predominam os Planossolos, Plintossolos, Espodossolos e Gleissolos (Fernandes et al., 2007).

A temperatura média anual varia entre 22 e $26^{\circ} \mathrm{C}$, e a precipitação média anual é de $1.400 \mathrm{~mm}$, com variação entre 800 e $1.600 \mathrm{~mm}$. Os maiores índices pluviais são observados no planalto. O período chuvoso estende-se de outubro a abril e concentra cerca de 70 a $80 \%$ da precipitação média anual.

A pecuária de corte extensiva de cria e recria é a atividade predominante no Pantanal; porém, em alguns casos, pode-se observar nas planícies mais elevadas, ao sul, leste e norte, pastagens plantadas utilizadas para engorda (Figura 1). Nesse bioma, a pastagem plantada é responsável por $98 \%$ das áreas que tiveram sua cobertura natural suprimida (Abdon et al., 2007).
Foram utilizados os dados meteorológicos de velocidade do vento, temperatura do ar e insolação coletados de estação meteorológica convencional, no Município de Corumbá, MS. Os dados meteorológicos foram obtidos do Banco de Dados Meteorológicos para Ensino e Pesquisa (BDMEP), do Instituto Nacional de Meteorologia (Inmet). Além disso, foram utilizados os dados dos produtos Mod09GA e Mod11A1, provenientes do sensor Modis/Terra do dia 11 de agosto de 2002.

No algoritmo Sebal, utiliza-se a equação do balanço de energia à superfície para estimar o fluxo de calor latente (LE) como um resíduo dos demais componentes do balanço de energia (Bastiaanssen et al., 1998a, 1998b; Allen et al., 2002): LE = Rn - H - G; em que Rn é o saldo de radiação ( $\mathrm{W} \mathrm{m}^{-2}$ ), $\mathrm{H}$ é o fluxo de calor sensível $\left(\mathrm{W} \mathrm{m}^{-2}\right)$, e $\mathrm{G}$ é o fluxo de calor no solo $\left(\mathrm{W} \mathrm{m}^{-2}\right)$.

Para o balanço de energia, o $\mathrm{Rn}$ foi a primeira variável a ser obtida. Para tanto, inicialmente, obteve-se o albedo da superfície conforme Liang (2001): $\alpha=0,160 \rho_{1}+0,291 \rho_{2}+0,243 \rho_{3}+0,116 \rho_{4}+0,112 \rho_{5}$ $+0,081 \rho_{7}-0,0015$; em que $\rho_{1}, \rho_{2}, \ldots, \rho_{7}$ são as bandas de reflectância do produto Mod09GA. Em seguida, foi estimado o saldo de radiação ( $R n)$ conforme Allen et al. (2002), por meio da equação $R n=R_{s \downarrow}-\alpha R_{s \downarrow}+$ $\mathrm{R}_{\mathrm{L} \downarrow}-\mathrm{R}_{\mathrm{L} \uparrow}-\left(1-\varepsilon_{\mathrm{o}}\right) \mathrm{R}_{\mathrm{L} \downarrow}$; em que $\mathrm{R}_{\mathrm{s} \downarrow}$ é a radiação de onda curta incidente na superfície ( $\mathrm{W} \mathrm{m}^{-2}$ ), $\alpha$ é o albedo da superfície (adimensional); $\mathrm{R}_{\mathrm{L} \downarrow}$ é a radiação de onda longa, emitida pela atmosfera na direção da superfície $\left(\mathrm{W} \mathrm{m}^{-2}\right) ; \mathrm{R}_{\mathrm{L} \uparrow}$ é a radiação de onda longa, emitida da superfície $\left(\mathrm{W} \mathrm{m}^{-2}\right)$; e $\varepsilon_{0}$ é a emissividade da superfície (adimensional). Portanto, na equação, o balanço de onda curta é função da radiação de onda curta incidente e do albedo da superfície, e o balanço de onda longa é quantificado em função do fluxo radiante de energia, emitido pelos gases atmosféricos e pelas superfícies líquidas e sólidas da Terra.

O fluxo de calor no solo $\left(\mathrm{G}, \mathrm{W} \mathrm{m}^{-2}\right)$ foi estimado conforme Bastiaanssen (2000),

$\mathrm{G}=\left[\mathrm{T}_{\mathrm{s}} / \alpha\left(0,0038 \alpha+0,0074 \alpha^{2}\right)\left(1-0,98 \mathrm{NDVI}^{4}\right)\right] \mathrm{Rn}$,

em que: $\mathrm{T}_{\mathrm{s}}$ é a temperatura da superfície $\left({ }^{\circ} \mathrm{C}\right)$, relativa ao produto Mod11A1, e NDVI ('normalized difference vegetation index') é o índice de vegetação da diferença normalizada. Para efeito de correção dos valores do fluxo de calor no solo, para corpos d'água (NDVI $<0$ ), considerou-se $\mathrm{G}=0,5 \mathrm{Rn}$ (Allen et al., 2002). Obtido o 
valor de $\mathrm{G}$, iniciou-se uma nova série de passos para a obtenção do fluxo de calor sensível $(\mathrm{H})$. Componente do balanço de energia, o $\mathrm{H}$ expressa a taxa de calor transferido da superfície para o ar pelos processos de convecção e condução. Esse fluxo é calculado pelo uso da velocidade do vento $(\mathrm{u})$, da resistência aerodinâmica da superfície $\left(\mathrm{r}_{\mathrm{ah}}\right)$ e da diferença da temperatura do ar (dT) entre dois níveis acima da superfície, nas alturas de 2,0 e 0,1 m. No Sebal, procedeu-se ao cálculo de dT para cada pixel, por meio de uma relação linear entre dT e Ts. Essa relação é dada pelos coeficientes a e b, obtidos dos pixels denominados âncoras, também chamados de quente e frio. Esses pixels-âncora foram selecionados mediante consulta simultânea às imagens de NDVI e temperatura da superfície (Ts). O pixel frio foi escolhido numa situação em que o valor do NDVI era negativo (geralmente sobre corpos d'água), com baixos valores de Ts, enquanto o pixel quente foi selecionado para a condição em que a Ts era elevada, e o NDVI inferior a 0,3 .

No pixel frio, as temperaturas da superfície e do ar próximo à superfície foram consideradas iguais. Neste
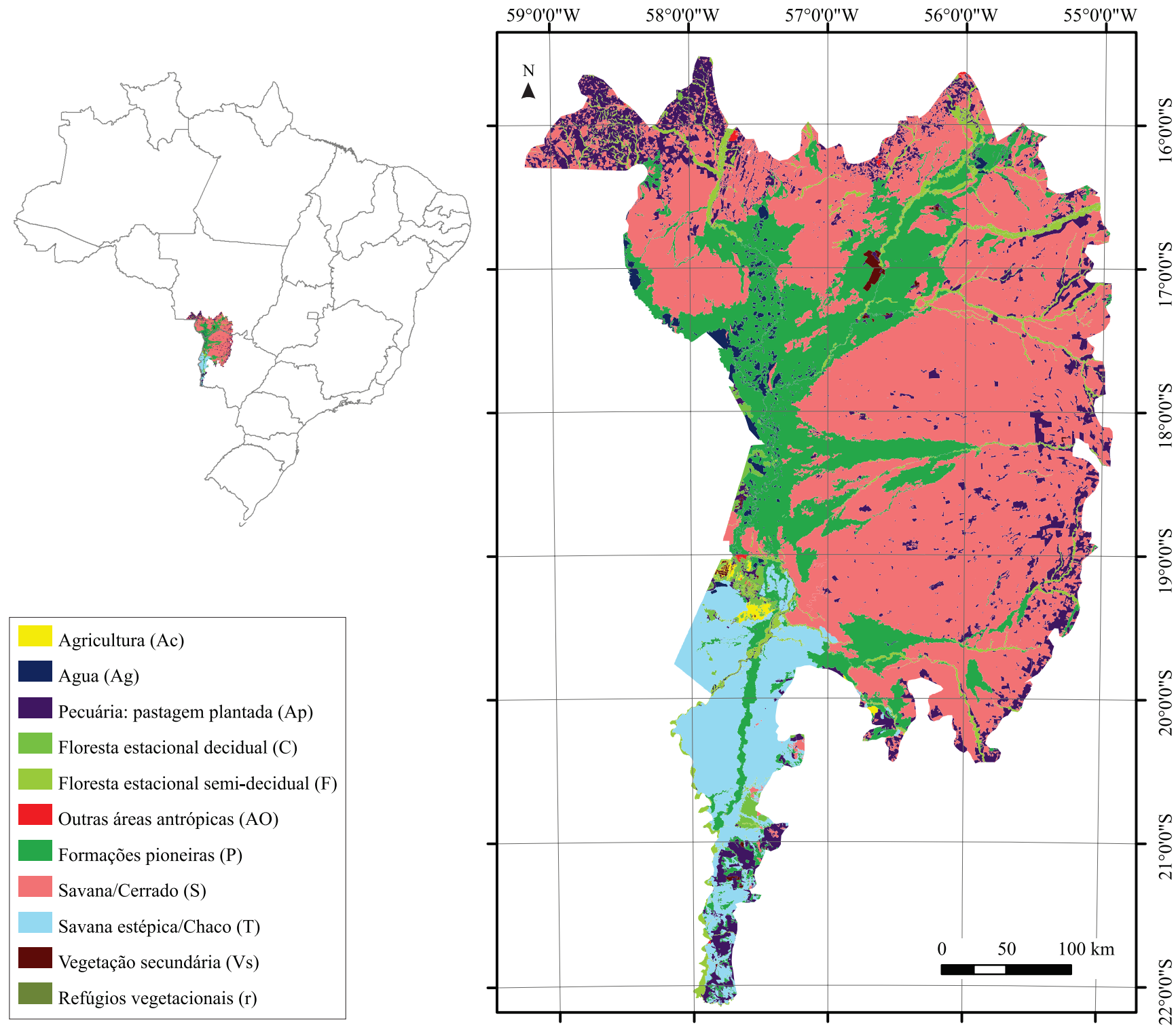

Figura 1. Localização do bioma Pantanal, com apresentação de classes de uso e cobertura da terra, provenientes do Programa de Conservação e Utilização Sustentável da Diversidade Biológica Brasileira (Probio).

Fonte: levantamento e mapeamento dos remanescentes da cobertura vegetal do bioma Pantanal, período de 2002 na escala de 1:250.000 (2004). 
caso, assumiu-se $\mathrm{H}$ igual a zero e determinou-se o LE máximo por meio da equação $L_{\text {frio }}=R n-G$. Para o pixel quente, assumiu-se LE igual a zero e obteve-se o H máximo pela equação $H_{\text {quente }}=R n-G=\rho c_{p}\left(a+b T_{s}\right) / r_{a h}$; em que a e b são parâmetros de ajuste, $\rho$ é a densidade do ar úmido $\left(\mathrm{kg} \mathrm{m}^{-3}\right), \mathrm{c}_{\mathrm{p}}$ é o calor específico do ar à pressão constante $\left(1.004 \mathrm{~J} \mathrm{~kg}^{1}{ }^{\circ} \mathrm{K}^{-1}\right)$, $\mathrm{r}_{\mathrm{ah}}$ é a resistência aerodinâmica e Ts é a temperatura da superfície (K). Por meio dos pixels-âncora, foi possível encontrar os coeficientes de correlação a e b para a obtenção de dT em cada pixel. Como no pixel frio dT é igual a 0 , ou seja, a + bTs $=0$, obteve-se um sistema com duas equações e duas incógnitas, o que possibilitou o cálculo de a e b. Logo, obteve-se também o fluxo de calor sensível inicial $\left(\mathrm{H}_{\text {inicial }}\right)$, por meio da expressão $\mathrm{H}_{\text {inicial }}=\rho c_{\mathrm{p}} \mathrm{dT} / \mathrm{r}_{\mathrm{ah}}$.

$\mathrm{Na}$ etapa seguinte, considerou-se condição de estabilidade atmosférica, por meio da realização de correções nos valores de $\mathrm{H}$ em processo iterativo. Para determinar essa condição, foi aplicada a teoria da similaridade de Monin-Obukhov (L, em m): se a atmosfera era instável, $\mathrm{L}<0$; se estável, $\mathrm{L}>0$; se neutra, $\mathrm{L}=0$. Os valores das correções de estabilidade para o transporte de momentum $\left(\psi_{\mathrm{m}}\right)$ e de calor sensível $\left(\psi_{\mathrm{h}}\right)$ foram obtidos conforme formulações sugeridas por Allen et al. (2002). Assim, foi possível corrigir a velocidade de fricção $\left(\mathrm{u}^{*}\right)$ e obter o valor corrigido de $\mathrm{r}_{\mathrm{ah}}$. Posteriormente, retornou-se ao cálculo da função da diferença de temperatura (dT) e repetiram-se os cálculos mencionados acima, até que se observasse estabilidade nos valores sucessivos de $\mathrm{dT}$ e $\mathrm{r}_{\mathrm{ah}}$ para $\mathrm{o}$ pixel quente. $\mathrm{O}$ passo seguinte foi determinar a fração evaporativa $\lambda=\mathrm{LE} /(\mathrm{LE}+\mathrm{H})=\mathrm{LE} /(\mathrm{Rn}-\mathrm{G})$; em que $\lambda$ é a fração evaporativa, definida como a fração de calor latente para o máximo saldo de energia disponível. Essa fração evaporativa pode ser considerada constante durante o período diurno, quando ocorre o processo de evapotranspiração (Crago, 1996). Assim, pode-se estimar a evapotranspiração real diária, por meio da equação proposta por Ayenew (2003): $\mathrm{ET}_{\text {diária }}=\lambda \mathrm{Rn}_{\text {diário }} / 28$, em que: $\mathrm{ET}_{\text {díria }}$ é a evapotranspiração real diária $\left(\mathrm{mm} \mathrm{d}^{-1}\right) ; \lambda$ é a fração evaporativa (adimensional); $\mathrm{Rn}_{\text {diário }}$ é o saldo de radiação integrado para um período de 24 horas, e o escalar no denominador representa o fator de conversão de unidades (de $\mathrm{W} \mathrm{m}^{-2}$ para $\mathrm{mm} \mathrm{d}^{-1}$ ).

Para a avaliação dos parâmetros biofísicos de superfície, de acordo com as classes de uso e cobertura da terra do Bioma Pantanal, foi utilizada a classificação do Programa de Conservação e Utilização Sustentável da Diversidade Biológica Brasileira (Probio), conforme Levantamento e mapeamento dos remanescentes da cobertura vegetal do Bioma Pantanal, período de 2002 na escala de 1:250.000 (2004).

Ao supor que o fluxo de calor no solo $(\mathrm{G})$, para o período de 24 horas, seja próximo de zero, foi possível estimar o fluxo de calor sensível diário $\left(\mathrm{H}_{\text {dário }}\right.$, em $\mathrm{W} \mathrm{m}^{-2}$ ) por meio da equação (Bastiaanssen, 2000): $\mathrm{H}_{\text {diário }}=(1-\lambda) \mathrm{Rn}_{\text {diário. }}$.

\section{Resultados e Discussão}

O uso e a cobertura da terra interferiram nos parâmetros avaliados (Figuras 2 a 5). Áreas de agricultura (Ac) apresentaram NDVI médio ao redor de 0,4 , valor que pode indicar que, no mês de agosto de 2002, algumas áreas de agricultura apresentavamse com vegetação seca ou exposição do solo, o que justifica os elevados valores médios de albedo $(0,15)$ e de temperatura da superfície (310 K) (Figura 2). Entre as classes avaliadas, o NDVI obtido para a área de abrangência da classe água (Ag) foi o que apresentou o maior desvio-padrão. A presença de vegetação pode ter sido o fator preponderante para tal resultado (Figuras 1 e 3 A).

Para as áreas de pecuária (Ap), o NDVI médio foi de $0,47 \pm 0,07$. Em relação à temperatura da superfície (Ts), o valor médio estimado foi de $309,0 \pm 1,8{ }^{\circ} \mathrm{K}$ (Figura 2). O maior desvio-padrão da Ts $\left(306,6 \pm 4,3^{\circ} \mathrm{K}\right.$ ) foi registrado para a área representada pela classe de savana estépica/chaco $(\mathrm{T})$, e o menor desvio-padrão da Ts $\left(308,8 \pm 1,45^{\circ} \mathrm{K}\right)$ foi obtido para a área delimitada pela classe referente a outras áreas antrópicas (OA). A pequena abrangência de área da classe OA (Figuras 1 e 3 B) pode ser uma das justificativas para o baixo desvio-padrão da Ts em comparação às demais classes (Figura 2). Em termos de abrangência, a classe savana/cerrado (S) é a de maior extensão do Bioma Pantanal. Na Figura 3 B, nota-se que, no cerrado, a Ts variou numa escala de valores intermediários e que, em boa parte da área, foram encontrados valores de Ts entre 306 e $310 \mathrm{~K}$.

$\mathrm{Na}$ classe água, o valor médio de albedo foi de $0,06 \pm 0,04$. Corpos d'água geralmente apresentam albedo entre 0,025 e 0,0348 (Allen et al., 2002). Nesse caso, além da vegetação, a escala do mapa de uso e cobertura da terra e a resolução espacial da imagem 
Modis são fatores que podem ter influenciado os resultados. Para áreas de campo alagado, Pereira et al. (2007) obtiveram albedo médio de 0,07 , em estimativas realizadas a partir de imagens TM-Landsat 5 (cenas de 30/9/2000, 16/2/2005, 4/3/2005 e 10/7/2005), na região de Passo do Lontra, Corumbá, MS.
Foram encontrados valores médios de albedo entre 0,11 e 0,13 , para as classes de floresta estacional decidual (C) e semidecidual (F), formações pioneiras (P), savana estépica/chaco e refúgios vegetacionais (r) (Figuras 1, 2 e 3 C). Nas classes de outras áreas antrópicas e de pecuária, o albedo médio foi de $0,16 \pm 0,02 \mathrm{e}$, para as classes de vegetação secundária
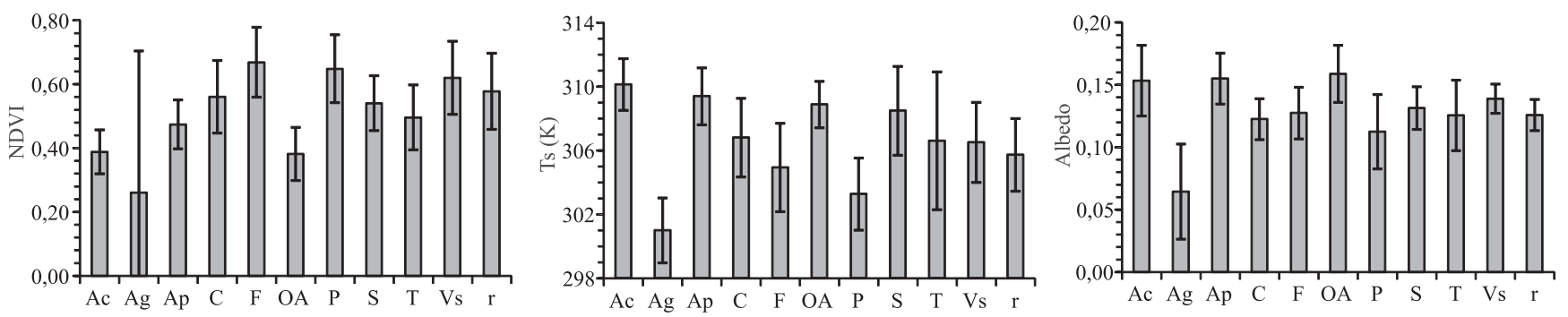

Figura 2. Valores médios com desvio-padrão do índice de vegetação da diferença normalizada (NDVI), temperatura da superfície (Ts, K) e albedo da superfície, para cada classe de uso e cobertura da terra no bioma Pantanal. Ac, agricultura; $\mathrm{Ag}$, água; Ap, pecuária; $\mathrm{C}$, floresta estacional decidual; F, floresta estacional semi-decidual; OA, outras áreas antrópicas; $\mathrm{P}$, formações pioneiras; S, savana/cerrado; T, savana estépica/chaco; Vs, vegetação secundária; r, refúgios estacionais.
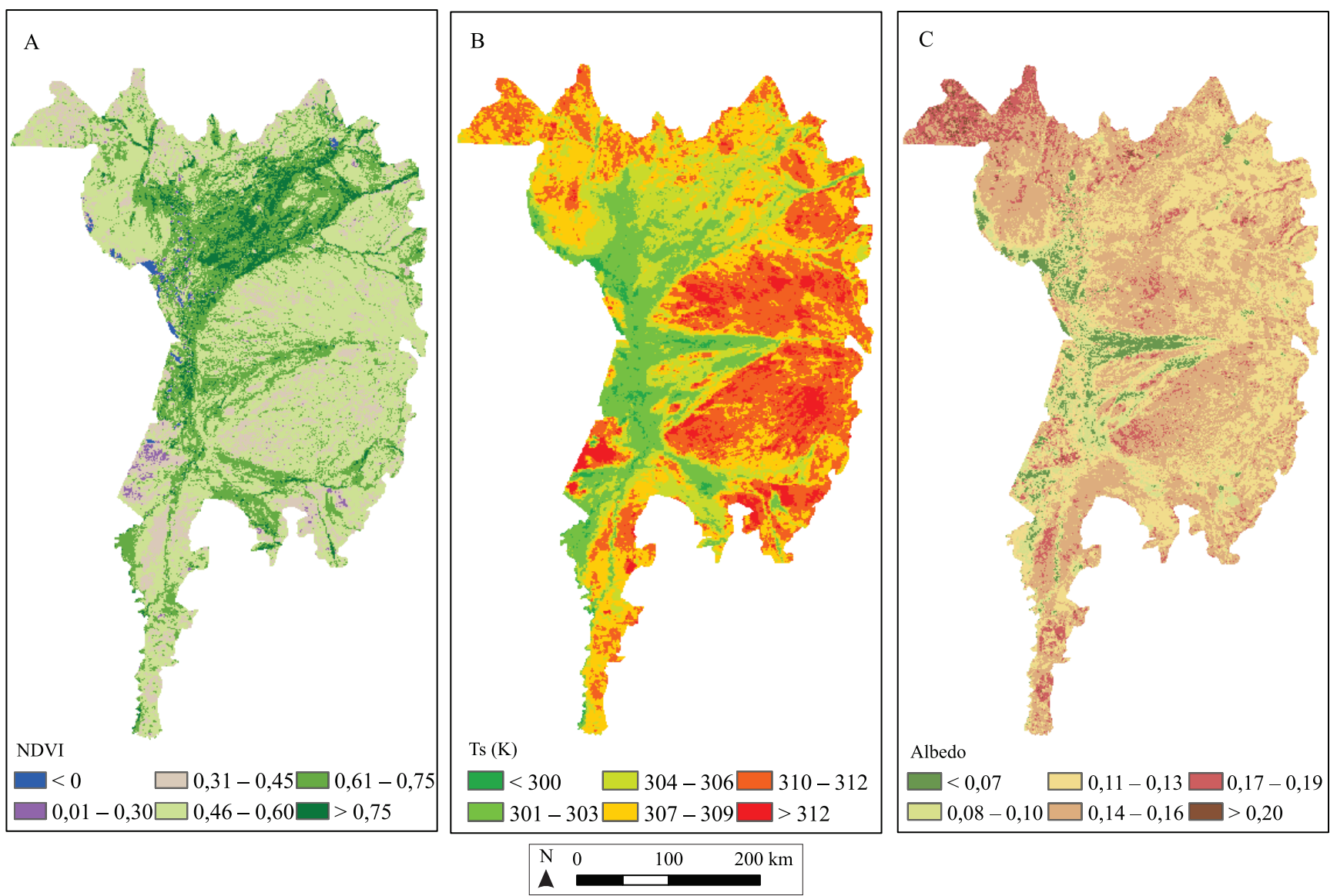

Figura 3. Mapas de: A, NDVI; B, temperatura da superfície (Ts); e C, albedo da superfície, para o bioma Pantanal. 
(Vs) e savana/cerrado, foi de $0,14 \pm 0,01$ e $0,13 \pm 0,02$, respectivamente. Observa-se que a classe pecuária destacou-se em termos espaciais, com valor de albedo acima de 0,16 em parte considerável da área de pecuária localizada na região norte do Pantanal (Figura 3 C).

Nas classes de agricultura e pecuária, foram observados os maiores valores de fluxo de calor sensível diário $\left(\mathrm{H}_{\text {diário }}\right)$. Nessas classes, a média do $\mathrm{H}_{\text {diário }}$ foi de
$42,0 \pm 6,4 \mathrm{~W} \mathrm{~m}^{-2}$ e $40,0 \pm 6,2 \mathrm{~W} \mathrm{~m}^{-2}$, respectivamente (Figuras 1, 4 e 5 A). Conforme esperado, os menores valores de $\mathrm{H}_{\text {dírio }}$ foram obtidos para a classe água. Nessa classe, o valor médio de $H_{\text {diário }}$ foi de $10,0 \pm 6,9$

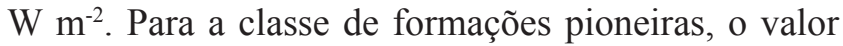
médio de $\mathrm{H}_{\text {diário }}$ foi de $19,0 \pm 8,0 \mathrm{~W} \mathrm{~m}^{-2}$; porém, na maior parte da área de abrangência dessa classe, os valores de $\mathrm{H}_{\text {diário }}$ variaram de 11 a $25 \mathrm{~W} \mathrm{~m}^{-2}$ (Figura $5 \mathrm{~A}$ ).
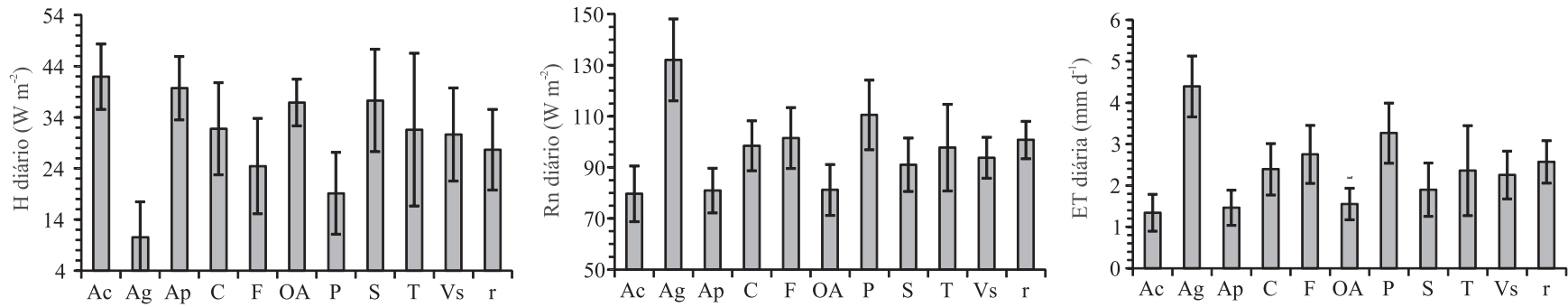

Figura 4. Valores médios com desvio-padrão de fluxo de calor sensível diário $\left(\mathrm{H}_{\text {diário }}, \mathrm{W} \mathrm{m}{ }^{-2}\right)$, saldo de radiação diário $\left(\mathrm{Rn}_{\text {diário, }}\right.$, $\left.\mathrm{W} \mathrm{m}{ }^{-2}\right)$, e evapotranspiração real diária $\left(\mathrm{ET}_{\text {diária }}, \mathrm{mm} \mathrm{d}^{-1}\right)$, para cada classe de uso e cobertura da terra no bioma Pantanal.
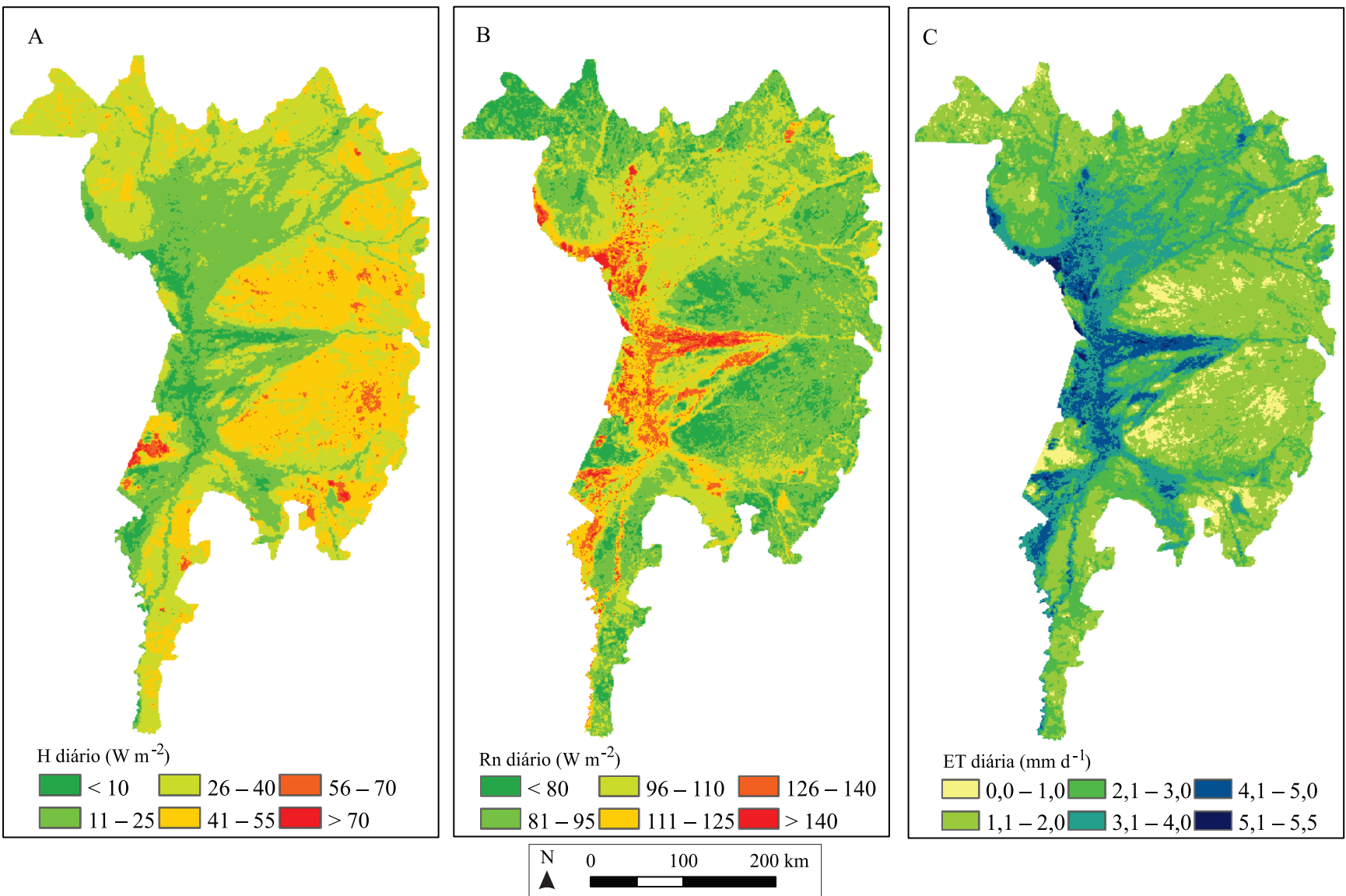

Figura 5. Mapas de: $\mathrm{A}$, fluxo de calor sensível diário $\left(\mathrm{H}_{\text {diário, }} \mathrm{W} \mathrm{m}^{-2}\right) ; \mathrm{B}$, saldo de radiação diário $\left(\mathrm{Rn}, \mathrm{W} \mathrm{m}^{-2}\right)$; e $\mathrm{C}$, evapotranspiração real diária (ET diária, $\mathrm{mm} \mathrm{d}^{-1}$ ), para o bioma Pantanal. 
Nas demais classes, a média de $\mathrm{H}_{\text {diário }}$ ficou entre 24 e $37 \mathrm{~W} \mathrm{~m}^{-2}$. No entanto, ressalta-se que valores de $\mathrm{H}_{\text {dírio }}$ acima de $40 \mathrm{~W} \mathrm{~m}^{-2}$ foram observados em boa parte da área referente à classe savana/cerrado. Sanches et al. (2011), ao avaliar os dados coletados de janeiro de 2007 a janeiro de 2008, em torre micrometeorológica instalada no Pantanal numa área sazonalmente inundada de cambará (Vochysia divergens), verificaram que a média do $\mathrm{H}_{\text {dírio }}$ foi de $25,46 \pm 10,4$ e $31,25 \pm 10,4$ $\mathrm{W} \mathrm{m}^{-2}$ para as estações seca e úmida, respectivamente. No entanto, para o mês de agosto, os autores obtiveram $\mathrm{H}_{\text {diário }}$ médio de $32,4 \pm 8,1 \mathrm{~W} \mathrm{~m}^{-2}\left(2,8 \pm 0,7 \mathrm{MJ} \mathrm{m}^{-2} \mathrm{~d}^{-1}\right)$. Em estudo realizado na fazenda São Bento (Corumbá, MS), a partir de dados micrometeorológicos coletados com auxílio de uma torre instalada numa área plana, constituída de gramíneas e ipês espaçados, com alturas de 8 a $10 \mathrm{~m}$, Oliveira et al. (2006) obtiveram valor médio de $\mathrm{H}_{\text {diário }}$ de $35 \mathrm{~W} \mathrm{~m}^{-2}$ para o período de setembro de 2000 a junho de 2002. Contudo, para um dia representativo da estação seca (4/9/2001), os autores observaram valor médio de $\mathrm{H}_{\text {dírio }}$ de $67,5 \mathrm{~W} \mathrm{~m}^{-2}$.

As áreas referentes às classes de pecuária, agricultura e outras áreas antrópicas apresentaram valores médios de saldo de radiação diário $\left(\mathrm{Rn}_{\text {diário }}\right)$ de cerca de $80 \mathrm{~W} \mathrm{~m}^{-2}$ (Figura 4). A classe água foi a que apresentou os maiores valores médios de $\operatorname{Rn}_{\text {dírio }}\left(132 \pm 16 \mathrm{~W} \mathrm{~m}^{-2}\right)$. No mapa da Figura $5 \mathrm{~B}$, nota-se $\mathrm{Rn}_{\text {diário }}$ acima de $100 \mathrm{~W} \mathrm{~m}^{-2}$, em áreas muito úmidas, alagadas ou de superfícies de corpos d'água. Em áreas de cambará (Vochysia divergens), sazonalmente inundadas, Sanches et al. (2011) observaram média mensal do $\mathrm{Rn}_{\text {diário }}$ de $106,5 \pm 21,9 \mathrm{~W} \mathrm{~m}^{-2}$, no mês de agosto, porém os autores obtiveram valores médios de 93,75 $\pm 33,6$ e $145,8 \pm 42,8 \mathrm{~W} \mathrm{~m}^{-2}$, para as estações seca e úmida, respectivamente.

A avaliação espacial do mapa na Figura 5 B permite notar que os valores do $\mathrm{Rn}_{\text {dírio }}$ variaram de 80 a $110 \mathrm{~W} \mathrm{~m}^{-2}$ em mais de $90 \%$ da área de abrangência da classe savana/cerrado. No entanto, a média do $\mathrm{Rn}_{\text {diário }}$ foi de $91 \pm 10 \mathrm{~W} \mathrm{~m}^{-2}$ (Figura 4). A partir de observações micrometeorológicas de uma área no Pantanal com vegetação típica de cerrado, Oliveira et al. (2006) constataram que a média do $\mathrm{Rn}_{\text {diário }}$ foi de $130 \mathrm{~W} \mathrm{~m}^{-2}$ nos meses de inverno.

Para as classes de agricultura, pecuária, outras áreas antrópicas e de savana/cerrado, foram encontrados valores médios da evapotranspiração real diária $\left(\mathrm{ET}_{\text {diária }}\right)$ entre 1,3 e 1,9 $\mathrm{mm} \mathrm{d}^{-1}$.
$\mathrm{Na}$ classe savana estépica/chaco, a média foi de $2,4 \mathrm{~mm} \mathrm{~d}^{-1}$ (Figura 4). No entanto, as estimativas realizadas para a área de abrangência dessa classe foram as que apresentaram o maior desvio-padrão da $\operatorname{ET}_{\text {diária }}\left(1,1 \mathrm{~mm} \mathrm{~d}^{-1}\right)$. Nas classes de formações pioneiras e água, foram observados valores médios acima de 3,0 $\mathrm{mm} \mathrm{d}^{-1}$. Para áreas de cambará (Vochysia divergens), sazonalmente inundadas, Sanches et al. (2011) observaram valores médios da $\mathrm{ET}_{\text {díria }}$ de

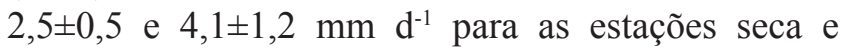
úmida, respectivamente. Além disso, para o mês de agosto, os autores observaram valor médio da $\mathrm{ET}_{\text {díria }}$ de $2,7 \pm 0,5 \mathrm{~mm} \mathrm{~d}^{-1}$.

A Figura $5 \mathrm{C}$ mostra a variação espacialmente explícita da $\mathrm{ET}_{\text {diária }}$ no Pantanal. Nota-se que a $\mathrm{ET}_{\text {diária }}$ variou de 4,0 a $5,5 \mathrm{~mm} \mathrm{~d}^{-1}$ em áreas do mapa com presença predominante de corpos d'água. Os valores de $\mathrm{ET}_{\text {diária }}$ entre 0,9 e 3,0 $\mathrm{mm} \mathrm{d}^{-1}$ foram observados para uma extensão considerável de área da classe savana/ cerrado.

Os resultados obtidos confirmam o potencial das geotecnologias como ferramentas para auxilio na implementação de modelos e algoritmos, voltados para a compreensão da complexa dinâmica dos processos biofísicos no âmbito da interação solo-planta-atmosfera no Pantanal.

\section{Conclusão}

A variação obtida de NDVI, temperatura da superfície, albedo, fluxo de calor sensível diário, saldo de radiação diário e evapotranspiração real diária, quanto aos diferentes usos e coberturas da terra, segue o padrão esperado e corrobora a eficiência da capacidade analítica e sinóptica das variáveis geradas pelo Sebal.

\section{Referências}

ABDON, M. de M.; SILVA, J. dos S.V. da; SOUZA, I. de M. e S.; ROMON, V.T.; RAMPAZZO, J.; FERRARI, D.L. Desmatamento no bioma Pantanal até o ano 2002: relações com a fitofisionomia e limites municipais. Revista Brasileira de Cartografia, n.59, p.17-24, 2007.

ADAMI, M.; FREITAS, R.M. de; PADOVANI, C.R.; SHIMABUKURO, Y.E.; MOREIRA, M. A. Estudo da dinâmica espaço-temporal do bioma Pantanal por meio de imagens MODIS.

Pesquisa Agropecuária Brasileira, v.43, p.1371-1378, 2008.

ALLEN, R.G.; TASUMI, M.; TREZZA, R.; WATERS, R.; BASTIAANSSEN, W. SEBAL: surface energy balance algorithms for land. Idaho implementation, advanced training and user's manual. Version 1.0. Idaho: University of Idaho, 2002. 98p. 
AYENEW, T. Evapotranspiration estimation using thematic mapper spectral satellite data in the Ethiopian rift and adjacent highland. Journal of Hydrology, v.279, p.83-93, 2003.

BASTIAANSSEN, W.G.M. SEBAL-based sensible and latent heat fluxes in the irrigated Gediz Basin, Turkey. Journal of Hydrology, v.229, p.87-100, 2000.

BASTIAANSSEN, W.G.M.; MENENTI, M.; FEDDES, R.A.; HOLTSLAG, A.A.M. A remote sensing surface energy balance algorithm for land (SEBAL): 1. Formulation. Journal of Hydrology, v.212-213, p.198-212, 1998a.

BASTIAANSSEN, W.G.M.; PELGRUM, H.; WANG, J.; MA, Y.; MORENO, J.F.; ROERINK, G.J.; WAL, T. van der. A remote sensing surface energy balance algorithm for land (SEBAL): 2. Validation. Journal of Hydrology, v.212-213, p.213-229, 1998 b.

COMPAORÉ, H.; HENDRICKX, J.M.; HONG, S.; FRIESEN, J.; GIESEN, N.C. van de; RODRIGUES, C.; SZARZYNSKI, J.; VLEK, P.L.G. Evaporation mapping at two scales using optical imagery in the White Volta Basin, Upper East Ghana. Physics and Chemistry of the Earth, v.33, p.127-140, 2008.

CRAGO, R.D. Conservation and variability of the evaporative fraction during the daytime. Journal of Hydrology, v.180, p.173-194, 1996.

FERNANDES, F.A.; FERNANDES, A.H.B.M.; SOARES, M.T.S.; PELLEGRIN, L.A.; LIMA, I.B.T. de. Atualização do mapa de solos da planície pantaneira para o sistema brasileiro de classificação de solos. Corumbá: Embrapa Pantanal, 2007. 6p. (Embrapa Pantanal. Comunicado técnico, 61).

LEVANTAMENTO e mapeamento dos remanescentes da cobertura vegetal do bioma Pantanal, período de 2002 na escala de 1:250.000. Campinas: Embrapa Informática Agropecuária, 2004. 43p.

LIANG, S. Narrowband to broadband conversions of land surface albedo. I. Algorithms. Remote Sensing of Environment, v.76, p.213-238, 2001.

OLIVEIRA, M.B.L. de; VON RANDOW, C.; MANZI, A.O.; ALVALÁ, R.C. dos S.; SÁ, L.D. de A.; LEITÃO, M. de M.V.B.R.; SOUZA, A. de. Fluxos turbulentos de energia sobre o Pantanal sul mato-grossense. Revista Brasileira de Meteorologia, v.21, p.159-165, 2006.

PEREIRA, G.; MORAES, E.C.; ARAI, E.; OLIVEIRA, L.G.L. Estudo preliminar da estimativa da reflectância e albedo de microssistemas pantaneiros a partir de imagens de satélite. Revista Brasileira de Cartografia, v.1, p.55-61, 2007.

SANCHES, L.; VOURLITIS, G.L.; ALVES, M. de C.; PINTO-JÚNIOR, O.B.; NOGUEIRA, J. de S. Seasonal patterns of evapotranspiration for a Vochysia divergens forest in the Brazilian Pantanal. Wetlands, v.31, p.1215-1225, 2011.

SANTOS, S.A.; COSTA, C. Manejo sustentável das pastagens nativas: uma ação essencial para a implantação de um sistema orgânico de produção no Pantanal. In: CONFERÊNCIA VIRTUAL GLOBAL SOBRE PRODUÇÃO ORGÂNICA DE BOVINOS DE CORTE, 1., 2002, Corumbá. Anais. Concórdia: University of Contestado; Corumba: Embrapa Pantanal, 2002. p.1-13. Disponível em: <www.cpap.embrapa.br/agencia/congressovirtual/ pdf/portugues/03pt07.pdf $>$. Acesso em: 30 ago. 2011.

TASUMI, M.; ALLEN, R.G.; TREZZA, R. At-surface reflectance and albedo from satellite for operational calculation of land surface energy balance. Journal of Hydrologic Engineering, v.13, p.51-63, 2008. 